\title{
Caracterización estructural del sistema de producción de alpacas (Vicugna pacos) en Huancavelica, Perú
}

\author{
Hinojosa, R.A. ${ }^{\circledR}$; Yzarra, A. ${ }^{2}$; Ruiz, J.A. ${ }^{3}$ y Castrejón, M. ${ }^{4}$
}

\begin{abstract}
'Oficina general de investigación. Universidad Nacional Autónoma de Chota. Cajamarca. Perú.
${ }^{2}$ Centro de producción de ciencias agrarias. Universidad Nacional de Huancavelica. Huancavelica. Perú.

${ }^{3}$ Laboratorio de biotecnología reproductiva. Universidad Nacional de Huancavelica. Huancavelica. Perú.

${ }^{4}$ Unidad de posgrado de la facultad de ingeniería. Universidad Nacional de Huancavelica. Huancavelica. Perú.
\end{abstract}

Palabras ClaVE ADICIONALES

\section{Manejo.}

Cría.

Encuesta.

Camélidos.

\section{ADDITIONAL KEYWORDS \\ Management. \\ Farming. \\ Inquiry. \\ Camelids.}

\section{INFORMATION}

\section{Cronología del artículo.}

Recibido/Received: 16.10.2017

Aceptado/Accepted: 04.09.2018

On-line: 15.01.2019

Correspondencia a los autores/Contact e-mail:

rhinojosab@unach.edu.pe

\section{RESUMEN}

El objefivo fue identificar los factores que ocasionan bajos resultados de explotación en el sistema de producción de alpacas del departamento de Huancavelica, en función del componente estructural, realizando un muestreo aleatorio estratificado con afijación proporcional. Se visitó 224 unidades productivas con alpacas (UPA) de más de 100 cabezas, analizando 40 variables, encontrando una dimensión media de 133,3 ha y 143,8 cabezas. El empadre es estacional de enero a marzo (37\%) y continuo todo el año (63\%), sin registros reproductivos idóneos (70\%). La hembra entra al primer empadre a los 22,6 meses en que su peso promedio bordea los $40 \mathrm{~kg}$ dentro de una relación de 11,3 hembras/macho, con alimentación a base de pastos naturales (100\%)

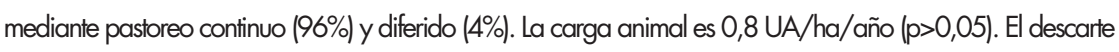
de machos y hembras se realiza a los $8,8 \pm 1,0$ años $(p \leq 0,05)$ y $7,9 \pm 0,7$ años $(p \leq 0,05)$, respectivamente. El tratamiento en salud animal se realiza en el $97 \%$ de las UPA, frente a una tasa de natalidad del $57,3 \pm 2,7 \%$ y peso al nacimiento $7,8 \pm 1,0 \mathrm{~kg}$; tasa de mortalidad de $5,6 \%$ en adultos y de $33,6 \%$ en crías. Los factores que ocasionan bajos resultados de explotación son: infraestructura inadecuada, instalaciones de manejo deficientes, ineficaz planificación de la reproducción, suplementación deficiente e ineficientes programas en salud animal.

\section{Structural characterization of the production system of alpacas Nicugna pacos) in Huancavelica, Peru}

\section{SUMMARY}

The aim of this study was to identify the factors that cause low exploitation results in the alpaca production system of the Huancavelica region, in function of the structural component, making a stratified random sampling with proportional affixation. Productive units with alpacas (PUA) (224) with more than 100 heads were visited, in which 40 variables were analyzed. The average size of PUA was 133.3 ha and 143.8 animals. The seasonal nesting is from January to March (37\%) and continuous feeding throughout the year (63\%) carry out reproduction and there are no ideal breeding records (70\%). The first mating takes place to female at the 22.6 months, with an average weight of $40 \mathrm{~kg}$. with a ratio of 11.3 females for every male. Feeding is based on natural pastures $(100 \%)$ by continuous grazing $(96 \%)$ and deferred $(4 \%)$ reaching a stocking density of $0.8 \mathrm{AU} /$ ha / year $(\mathrm{P}>$ 0.05 ). Discarding males and females was performed at $8.8 \pm 1.0$ years $(P \leq 0.05)$ and $7.9 \pm 0.7$ years $(P \leq 0.05)$, respectively. The PUA, although $97 \%$ of them apply preventive treatments. The birth rate $57.3 \pm 2.7 \%$. The birth weight $7.8 \pm 1.0 \mathrm{~kg}$. The mortality rate $5.6 \%$ in adults and $33.6 \%$ in offspring. The factors that cause low ope rating results are: inadequate infrastructure, poor management facilities, inefficient reproductive planning, poor supplementation, and inefficient animal health programs.

\section{INTRODUCCIÓN}

El Perú es el primer referente en la crianza de alpacas, concentrando el $80 \%$ de la población mundial. A nivel nacional la población actual es de 3685 516, según el IV Censo Nacional Agropecuario CENAGRO-2012.
Las alpacas representan un recurso importante para la producción de carne en las familias de la zona altoandina (Fairfield 2006, p.32).

La vida productiva promedio en alpacas hembra es de siete años y en machos de cinco, siendo el período que permanecen como productores de fibra (Laura 
2015, p.136). En el departamento de Huancavelica la población es de 308586 cabezas, es decir el 8,37\% de la población nacional, de las cuales 255472 corresponden a la raza Huacaya, 12278 a la raza Suri y 34857 a las alpacas de raza primaria cruzadas con la raza estandarizada, adicionalmente 5979 capones, destacando la provincia de Huancavelica con 129737 cabezas (INEI 2012, p.47). En el plano regional, cuatro de las siete provincias involucran el potencial alpaquero de la zona y estas son Angaraes, Castrovirreyna, Huancavelica y Huaytará, constituyendo el corredor económico central regional de los camélidos sudamericanos donde se desenvuelven alrededor de 18 000 familias en torno a esta actividad económica (Buritica 2010, p.13). Las unidades productivas con alpacas (UPA) en el Perú son en su mayoría comunitarias. La ganadería altoandina se basa generalmente en el pastoreo de campos nativos liderados por productores de escasos recursos económicos, quienes realizan una crianza de baja escala técnica (Aguirre et al. 2011, p.48), actualmente desarrollan una mala práctica de manejo, que es la quema de los pastizales, produciendo de esta manera un deterioro de las praderas (Mendoza 2007, p.34). Las UPA son sustento de los pobladores alto-andinos, generan fuente de: alimento (carne), vestido (fibra, piel), abono para la tierra (heces), combustible (heces secas) y animal de carga. Es conocido que la carne tiene buenos parámetros tecnológicos de calidad (Saadoun \& Cabrera 2008 p.579; Salvá et al. 2009, p.450). Los camélidos sudamericanos domésticos son un recurso genético nativo de alto valor socioeconómico en la zona alto-andina; sin embargo, la condición actual de los sistemas productivos asociados con esta especie no permite identificarlos como elementos impulsores de una mejora sustantiva de los medios de subsistencia de sus productores (Quispe et al. 2009b, p.11). La caracterización estructural permite determinar el nivel tecnológico implementado por los sistemas productivos estudiados, relacionado con los diferentes aspectos que recaen en el proceso productivo (infraestructura, reproducción, alimentación y salud, entre otros) (Valerio 2010, p.334). Por estas razones, el presente trabajo se llevó a cabo con el objetivo de identificar los factores que causan bajos resultados de explotación en el sistema de producción de alpacas del departamento de Huancavelica.

\section{MATERIALES Y MÉTODOS}

El estudio se desarrolló en las provincias de Angaraes, Castrovirreyna, Huancavelica, y Huaytará, pertenecientes al departamento de Huancavelica. Es- tas provincias comprenden 755 UPA y 32217 cabezas, constituyendo las principales zonas de producción de alpacas del departamento de Huancavelica. En la tabla I se presentan informaciones de localización geográfica, datos climáticos y zonas de vida de las provincias que conforman la zona de estudio. Se realizó un muestreo aleatorio estratificado con afijación proporcional al tamaño del estrato (Santos et al. 2004, p.170). Se recolectó la información entre marzo y junio de 2016 mediante encuestas directas con el productor alpaquero, aplicando un formulario de encuesta estructurado por 100 preguntas cerradas, que recogió aspectos generales y dimensión productiva del productor (03), infraestructura e instalaciones (32), aspectos reproductivos (25), alimentación (23), intensificación y estructura del rebaño (04), sanidad (13). Dicha información se complementó con fotos de las instalaciones de cada UPA, de acuerdo con la metodología utilizada por Milán et al. Esta metodología está en concordancia con la utilizada por Cochi et al. (2014, p.122). Se recolectó la información entre marzo y junio de 2016 mediante encuestas directas con el productor alpaquero, aplicando un formulario de encuesta estructurado por 100 preguntas cerradas, que recogió aspectos generales y dimensión productiva del productor (03), infraestructura e instalaciones (32), aspectos reproductivos (25), alimentación (23), intensificación y estructura del rebaño (04), sanidad (13). Dicha información se complementó con fotos de las instalaciones de cada UPA, de acuerdo con la metodología utilizada por Castaldo et al. (2006, p.184).

El marco poblacional estuvo constituido por las UPA con más de 100 cabezas, es decir 755 UPA, según censo agropecuario (INEI 2012, p.47), seleccionándose una muestra de 224 UPA. El nivel de representatividad de la muestra se presenta en la Tabla II y Figura 1.

Las UPA se estratificaron por provincia (Angaraes, Castrovirreyna, Huancavelica y Huaytará) y la especie animal alpaca. Se analizó y codificó 40 variables representativas de la dimensión productiva, infraestructura, instalaciones, reproducción, alimentación, intensificación y sanidad. Se empleó el software SPSS Statistics 17.0.2 para el análisis de varianza de las variables cuantitativas y el test de tukey-kramer para la prueba de comparaciones múltiples. Las variables categóricas fueron analizadas mediante tablas de frecuencias y el test Chi cuadrado. Esta metodología de análisis va de acuerdo con lo implementado por Gaspar (2009, p.121).

Tabla I. Localización geográfica y datos climáticos de la zona de estudio (Geographical location and climatic data of the study área).

\begin{tabular}{|c|c|c|c|c|c|c|}
\hline Provincia & Zona de vida * & Latitud & Longitud & $\mathrm{P}$ & $\mathrm{T}$ & $\mathrm{H}$ \\
\hline Angaraes & md-MBS & $12^{\circ} 59^{\prime} 00^{\prime \prime} \mathrm{S}$ & $74^{\circ} 43^{\prime} 03^{\prime \prime} \mathrm{W}$ & 828.2 & 13.0 & 82 \\
\hline Castrovirreyna & $\mathrm{pp}-\mathrm{ST}$ & $13^{\circ} 16^{\prime} 54^{\prime \prime} \mathrm{S}$ & $75^{\circ} 19^{\prime} 04^{\prime \prime} \mathrm{W}$ & 680.0 & 6.3 & 82 \\
\hline Huancavelica & $\mathrm{pp}-\mathrm{ST}$ & $12^{\circ} 47^{\prime} 15^{\prime \prime} \mathrm{S}$ & $74^{\circ} 58^{\prime} 23^{\prime \prime} \mathrm{W}$ & 897.5 & 9.0 & 86 \\
\hline Huaytará & pp-ST & $13^{\circ} 36^{\prime} 10^{\prime \prime} \mathrm{S}$ & $75^{\circ} 21^{\prime} 11^{\prime \prime} \mathrm{W}$ & 685.3 & 13.1 & 77 \\
\hline \multicolumn{7}{|c|}{$\begin{array}{l}\text { Fuente: IGN, } 1999 \text { y PER-HVCA, 2009-2015. } \\
\text { P= precipitación promedio anual (mm) } \\
\text { T= temperatura media anual (pc) } \\
\mathrm{H}=\text { humedad relativa media anual }(\%) \\
\text { * = Sistema de Clasificación de Zonas de Vida (Holdridge, 1967) }\end{array}$} \\
\hline
\end{tabular}




\section{RESULTADOS Y DISCUSIÓN}

\section{ASPECTOS GENERALES Y DE DIMENSIÓN}

La UPA es una empresa familiar en el 100\% de los casos; similar a lo reportado por Celorio et al. (2011, p.409) quienes con sus encuestas mostraron que en la estructura de las granjas de ganado el $86,7 \%$ son negocios familiares; coincidiendo con Sulbarán et al. (2008, p.82) quien afirmó que, en un sistema de producción con ganado, la fuerza de trabajo más importante es familiar.

Los pastores de alpacas no tienen educación secundaria, mucho menos conocimientos técnicos para dar valor agregado a sus productos; similar a lo informado por Laura (2015, p.135) quien afirmó que el nivel educativo de los productores de alpaca se enmarca en un $68 \%$ con instrucción primaria, $28 \%$ con educación secundaria incompleta y el resto sin ninguna educación; cercano a lo reportado por Valerio et al. (2009, p.639) quienes habían indicado que entre el 60 y el $65 \%$ de los productores de ovino iniciaron los estudios primarios, aunque una gran proporción de ellos no los completó; esto en concordancia con Cárdenas (2013, p.6) quien encontró que el 52,2\% de los productores de Santa Ana, Huancavelica tienen educación primaria (completa e incompleta) y solo el 5,8\% han completado la educación superior; coincidiendo también con Parodi (2011, p.28) quien indicó que los pocos conocimientos en técnicas adecuadas como esquila, acopio y clasificación para la calidad de la fibra, limitan y dificultan las confecciones de prendas de vestir de alpaca de buena calidad para comercializarlas.

La edad promedio del propietario gira en torno a los 54,1 \pm 2,7 años; ligeramente por encima de lo reportado por Laura (2015, p.135) quien determinó que la edad promedio de los alpaqueros de Pacajes es de 52 años; similar a lo informado por Celorio et al. (2011, p.409) quienes reportaron que el 73,3\% de los productores de ganado son mayores de 50 años; coincidiendo con Cárdenas (2013, p.6) quien aseveró que 56,5\% de productores de Alaska en Santa Ana tienen más de 50 años; en concordancia con Gonzálvez (2010, p.47) quien concluyó que los productores de edad avanzada, acostumbrados a una forma de trabajar durante toda su vida, no contemplan la adaptación a nuevas exigencias; coincidiendo también con Valerio et al. (2009, p.643) quienes concluyeron que entre los factores que limitan la sostenibilidad de los sistemas de producción

\begin{tabular}{llll}
\hline \multicolumn{4}{l}{ Tabla II. Distribución y representatividad de la } \\
muestra (Distribution and representativeness of the sample). \\
\hline Provincia & $\mathrm{N}$ & $\mathrm{n}$ & $\mathrm{N}^{\circ}$ de cabezas \\
\hline Angaraes & 61 & 18 & 2349 \\
Castrovirreyna & 65 & 19 & 2061 \\
Huancavelica & 380 & 113 & 19304 \\
Huaytará & 249 & 74 & 8503 \\
\hline Total & 755 & 224 & 32217
\end{tabular}

Fuente: Instrumento de investigación aplicado a productores alpaqueros de las unidades productivas con alpacas de Huancavelica, May 2016

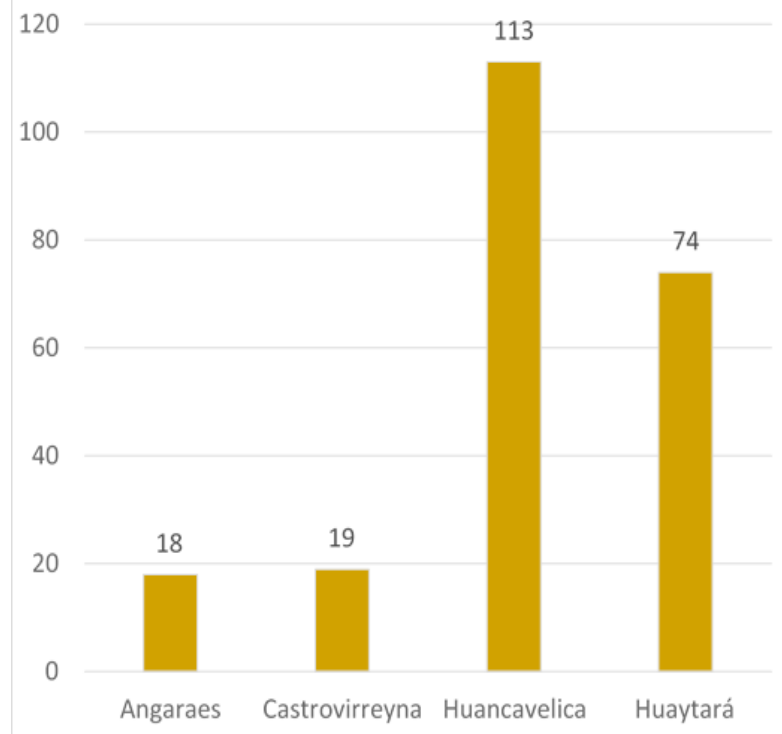

Figura 1. Distribución de frecuencias del número de UPA que tienen más de 100 cabezas, seleccionadas como muestra (Frequency distribution of UPA number having more than 100 heads, selected as sample).

de cabras está la edad avanzada de los productores, alrededor de los 51 años.

El uso de la tierra es de propiedad comunal (96\%) (Tabla IV); ligeramente por encima de lo reportado por Gómez (2013, p.39) quien indicó que el 90,7\% del sistema productivo de cabras en Apurímac se desarrolla dentro del régimen de tenencia comunal de la tierra.

Existe un sistema de manejo extensivo en las UPA de Huancavelica; coincidiendo con Aguirre et al. (2011, p.12) quien afirmó que en el sector alpaquero peruano los sistemas de producción son extensivos, basados en el pastoreo de campos nativos de manejo tradicional, con una mínima técnica artesanal de cría; tal como lo mencionó Quina (2015, p.34) quien afirmó que el rancho ganadero andino generalmente se desarrolla extensivamente, ya que depende de los pastizales que existen en las praderas naturales; de acuerdo también con lo reportado por Gómez $(2013$, p.39) quien afirmó que el sistema productivo que caracteriza a las granjas caprinas de la región Apurímac, es extensivo.

Se encontró una dimensión promedio de 133,4 \pm 68,5 ha y $143,8 \pm 45,3$ alpacas $(\mathrm{P} \leq 0.05)$ (Tabla III), con un coeficiente de variación de 51,5 and $31,5 \%$, respectivamente; También se muestra la existencia de diferencias significativas $(\mathrm{P} \leq 0,05)$ al enfrentar la dimensión respecto a la especie y la provincia.

\section{ASPECTOS DE INFRAESTRUCTURA E INSTALACIONES}

En términos de infraestructura, el 76\% de las UPA presentan inadecuadas y difíciles vías de acceso (Tabla IV) y el $87 \%$ carecen de energía eléctrica; coincidiendo en parte con Aguirre et al. (2011, p.49) quien indicó que el sector alpaquero peruano tiene una infraestructura vial deficiente, así como una infraestructura de riego precario, mientras que el 98\% tiene telefonía móvil, ya que actualmente hay un aumento considerable en 
Tabla III. Características estructurales y productivas de las UPA de cuatro provincias del departamento de Huancavelica (Structural and productive characteristics of the UPA in four provinces of the department of Huancavelica)

\begin{tabular}{|c|c|c|c|c|c|c|}
\hline \multicolumn{7}{|c|}{ Unidades Productivas con Alpacas n=224 } \\
\hline & $(n=18)$ & $(n=19)$ & $(n=113)$ & $(n=74)$ & & \\
\hline Variables & 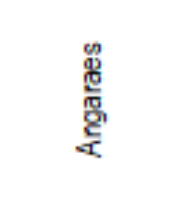 & 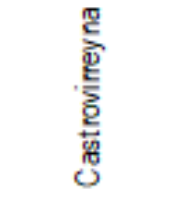 & 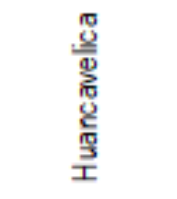 & 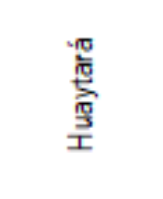 & $X \pm E S$ & $c v \%$ \\
\hline Ed ad de l propietario (años) & $51,7 \pm 2,4^{a}$ & $53,6 \pm 2,5^{a}$ & $55,6 \pm 2,1^{\circ}$ & $52,6 \pm 2,2^{2}$ & $54,1 \pm 2,7$ & 4,9 \\
\hline Nume ro de cabezas/UA & $130,5 \pm 25,8^{a}$ & $108,5 \pm 25,7^{a}$ & $170,8 \pm 47,8^{\circ}$ & $114,9 \pm 8,8^{a}$ & $143,8 \pm 45,3$ & 31,5 \\
\hline Carga animal (UA/ha/año) & $0,8 \pm 0,1^{\mathrm{a}}$ & $0,7 \pm 0,2^{2}$ & $0,8 \pm 0,1^{a}$ & $0,7 \pm 0,2^{2}$ & $0,8 \pm 0,1$ & 18,6 \\
\hline Ed ad de instalaciones (años) & $20,2 \pm 2,4^{a}$ & $19,2 \pm 1,4^{a}$ & $39,2 \pm 2,4^{\circ}$ & $31,1 \pm 2,4^{c}$ & $33,3 \pm 7,4$ & 22,3 \\
\hline Ed ad de ste te (meses) & $8,4 \pm 0,8^{a}$ & $7,9 \pm 0,8^{\circ}$ & $7,1 \pm 0,4^{c}$ & $8,4 \pm 0,6^{9}$ & $7,7 \pm 0,8$ & 10,1 \\
\hline Edad machos primer e mpadre (meses) & $31,4 \pm 1,5^{a}$ & $33,4 \pm 1,5^{\circ}$ & $32,4 \pm 1,5^{50}$ & $32,4 \pm 2,5^{20}$ & $32,4 \pm 1,9$ & 5,9 \\
\hline Ed ad hemb ras primer e mpadre (meses & $24,8 \pm 1,1^{a}$ & $24,8 \pm 1,4^{a}$ & $21,8 \pm 2,4^{\circ}$ & $22,8 \pm 1,2^{c}$ & $22,6 \pm 2,2$ & 9,6 \\
\hline Edad de scarte hembras (años) & $8,9 \pm 0,3^{a}$ & $8,1 \pm 0,8^{604}$ & $7,6 \pm 0,5^{c}$ & $8,1 \pm 0,7^{\square}$ & $7,9 \pm 0,7$ & 8,8 \\
\hline Ed ad de scare machos (años) & $9,4 \pm 0,5^{a}$ & $9,4 \pm 0,9^{2}$ & $8,4 \pm 1,2^{0}$ & $9,2 \pm 0,5^{a}$ & $8,8 \pm 1,0$ & 11,6 \\
\hline Re lación hembra/macho & $11,6 \pm 1,2^{a}$ & $11,3 \pm 1,6^{2}$ & $11,2 \pm 1,6^{a}$ & $11,4 \pm 1,5^{a}$ & $11,3 \pm 1,5$ & 13,4 \\
\hline Tasa de natalidad $(\%)$ & $55,6 \pm 2,3^{a}$ & $56,6 \pm 2,2^{2}$ & $58,7 \pm 2,4^{\circ}$ & $55,8 \pm 2,2^{2}$ & $57,3 \pm 2,7$ & 4,7 \\
\hline Tasade fertildad ( $\%$ & $71,5 \pm 1,8^{\mathrm{a}}$ & $70,1 \pm 2,4^{a}$ & $78,5 \pm 1,2^{c}$ & $73,5 \pm 2,5^{a}$ & $75,6 \pm 3,6$ & 4,7 \\
\hline Pe so al nacimiento $(\mathrm{kg})$ & $7,1 \pm 0,9 \mathrm{a}$ & $6,4 \pm 0,5^{\circ}$ & $8,4 \pm 0,7^{c}$ & $7,3 \pm 0,9 \mathrm{a}$ & $7,8 \pm 1,0$ & 13,1 \\
\hline Pe so al de ste te $(\mathrm{kg})$ & $24,1 \pm 0,7^{x}$ & $23,9 \pm 0,9^{2}$ & $25,9 \pm 0,9^{\circ}$ & $24,7 \pm 0,9^{C}$ & $25,2 \pm 1,1$ & 4,5 \\
\hline Superficie to tal (ha) & $51,7 \pm 38,3^{a}$ & $57,4 \pm 22,4^{a}$ & $184,8 \pm 49,9^{\circ}$ & $94,3 \pm 35,6^{c}$ & $133,4 \pm 68,5$ & 51,4 \\
\hline Infertidad de hembras $(\%)$ & $8,7 \pm 0,8^{x}$ & $9,6 \pm 0,5^{\circ}$ & $7,2 \pm 0,5^{a}$ & $8,2 \pm 1,3^{c}$ & $7,9 \pm 1,1$ & 14,6 \\
\hline Infertidad de machos (\%) & $4,2 \pm 0,6^{2}$ & $4,2 \pm 0,4^{a}$ & $3,1 \pm 0,3^{0}$ & $3,9 \pm 0,8^{a}$ & $3,5 \pm 0,7$ & 19,9 \\
\hline Mortalidad de crias $(\%)$ & $38,2 \pm 2,3^{a}$ & $37,2 \pm 2,0^{x}$ & $31,2 \pm 3,8^{\circ}$ & $35,2 \pm 2,0^{c}$ & $33,6 \pm 4,0$ & 11,9 \\
\hline Mortalidad de adultos ( $\%$ & $6,5 \pm 1,4^{2}$ & $5,5 \pm 0,5^{20}$ & $5,3 \pm 1,5^{b c}$ & $5,9 \pm 1,4^{x}$ & $5,6 \pm 1,4$ & 25,5 \\
\hline Nume ro de instal aciones & $2,2 \pm 0,4^{200}$ & $2,1 \pm 0,2^{a}$ & $2,4 \pm 0,5^{0}$ & $2,3 \pm 0,4^{a}$ & $2,3 \pm 0,5$ & 21,0 \\
\hline
\end{tabular}

$X \pm E S$, letras diferentes en la misma fla indican diferencias significativas $(p \leq 0,05)$

el uso de servicios de comunicación, principalmente teléfonos móviles que facilitan un mayor acceso a canales de comercialización de insumos, productos y servicios. El 100\% del suministro de agua en la UPA de Huancavelica proviene de arroyos y manantiales, también llamados humedales altoandinos (HAA) o "bofedales", que pertenecen a un tipo de ecosistema que se caracteriza por tener vegetación perenne dentro del paisaje semi-árido de las zonas altoandinas; coincidiendo con Laura $(2015$, p.80) quien afirmó que las comunidades alpaqueras se proveen de agua proveniente de laderas, condiciones que permiten un mayor riego de sus "bofedales"; de acuerdo con lo informado por Mendoza $(2007$, p.34) quien afirmó que el agua que consumen los pequeños rumiantes y camélidos proviene de ojonales, laderas y "bofedales"; similar a lo reportado por García y Otto (2015, p.116), Squeo et al. (2006, p.245) quienes indicaron que el agua subterránea originada de las lluvias y el deshielo de los glaciares y la fusión de nieve son la principal fuente de agua de estos HAA. El 100\% de UPA no utiliza instalaciones para el manejo de las crías, lo que conlleva a su mortalidad debido a la incidencia de enfermedades y el acoso de los depredadores, principalmente zorros; en este sentido, el $60 \%$ de las UPA estudiadas carecen de cercos perimétricos, observándose diferencias significativas entre ellas $(\mathrm{P} \leq 0.05)$ (Tabla IV).

Las instalaciones utilizadas en las UPA de Huancavelica son rústicas construidas con materiales de la zona como piedra, barro e ichu (Figura 2), con una edad promedio de 33,3 \pm 7,4 años (Tabla III). Cárdenas $(2013$, p.6) reportó que el $75 \%, 10.3 \%, 98.5 \%, 7.4 \%$ y $17.6 \%$ de los productores tiene cobertizo, corrales de empadre, bañadero comunal, corrales de manejo y canchas de descanso para el pastoreo en época seca respectivamente, en Santa Ana, Huancavelica; muy cercano a lo reportado por Carné et al. (2007, p.43), quien al realizar la caracterización estructural de cabras reportó que las instalaciones son corrales de montaña en mal estado; en consonancia con Vargas (2007, p.64) quien afirmó que al caracterizar la producción ganadera de Pacajes, en términos de infraestructura bovina, los rebaños bovinos no cuentan con una infraestructura diseñada en ninguno de los casos. El ganado duerme 


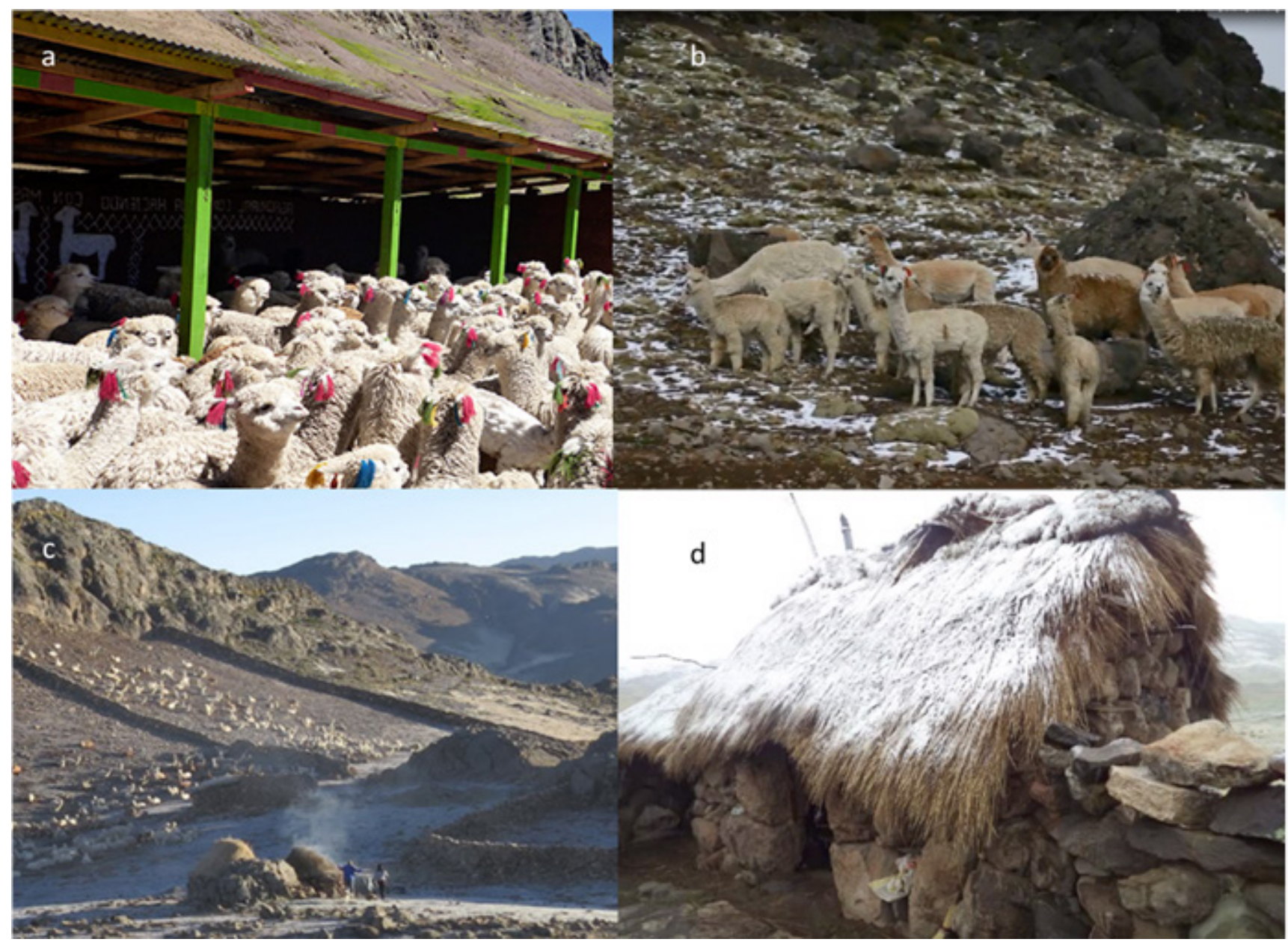

Figura 2. (a) cobertizo en la provincia de Huancavelica; (b) alpacas pastando en la provincia de Huaytará; (e) alpacas en la provincia de Castrovirreyna; (d) una choza., en la provincia de Angaraes (a) shed in the province of Huancavelica; (b) Alpacas grazing in the province of Huaytara; (e) Alpacas in the province of Castrovirreyna; (d) A "hut., in the province of Angaraes).

en el campo libre, simplemente amarrado en estacas, para que no escapen en las noches, basado en el conocimiento ancestral rudimentario; ya Mendoza (2007, p.35) había declarado que los pequeños rumiantes y camélidos estaban alojados en una infraestructura de corrales de manejo precario, observando solo corrales que no garantizan la protección de los animales de las condiciones climáticas extremas, como la presencia de heladas, lluvia y nieve.

\section{MANEJO REPRODUCTIVO}

Predomina el uso de la monta natural continua (63\%) (Tabla IV), con empadres y partos durante todo el año debido principalmente al manejo continuo de los machos juntos con las hembras que permanecen en celo continuo, sin registros de producción y reproducción; tal como lo mencionó Mendoza $(2007$, p.32) que los productores de alpacas no manejan por separado los machos y las hembras, estos pasan juntos durante todo el año reproduciéndose sin ninguno tipo de control dando origen a la consanguinidad lo que se refleja en la producción; contrariamente a lo indicado por Allende $(2008$, p.21) quien afirma que el momento de la cría y el parto comienza casualmente con el comienzo de las lluvias, y los animales pueden estar en un estado nutricional de transición que influirá en la fertilidad masculina y femenina.

La Tabla III muestra que la edad del macho al primer empadre es a los $32,4( \pm 1,9)$ meses $(\mathrm{P} \leq 0,05)$, de la hembra a los $22,6( \pm 2,2)$ meses $(\mathrm{P} \leq 0,05)$ que oscila entre los 18 y 27 meses. El empadre de las hembras bajo condiciones de pastoreo en pasturas naturales se realiza a los dos años de edad, en contraste con lo reportados por Hinojosa y Ruiz (2003, p.750) quienes en base a observaciones y estudios determinaron que la tasa de natalidad en alpacas empadradas al año de edad es similar a la de las adultas, demostrándose así mismo que las hembras que alcanzan el 60\% (35 kg.) de su peso adulto se reproducen normalmente; contradiciendo en parte a Mendoza (2007, p.34) quien indicó que el método reproductivo utilizado es la monta natural, la edad reproductiva tanto del macho como de la hembra inicia a los 12 meses, por lo que las hembras no alcanzan el desarrollo corporal adecuado para iniciar la etapa reproductiva lo que se traduce en la disminución en la producción. La relación hem$\mathrm{bra} /$ macho es de $11,3( \pm 1,5)(\mathrm{p}>0,05)$, relación que se asemeja a lo reportado por Cárdenas (2013) quien recomendó una relación de 1:10 en este tipo de sistema extensivo. La edad media de descarte de machos es a 
Tabla IV. Infraestructura, pastoreo, reproducción, alimentación y sanidad en las UPA de cuatro provincias del departamento de Huancavelica (Infrastructure, grazing, reproduction, food and sanitation in the UPA of four provinces of the Department of Huancavelica).

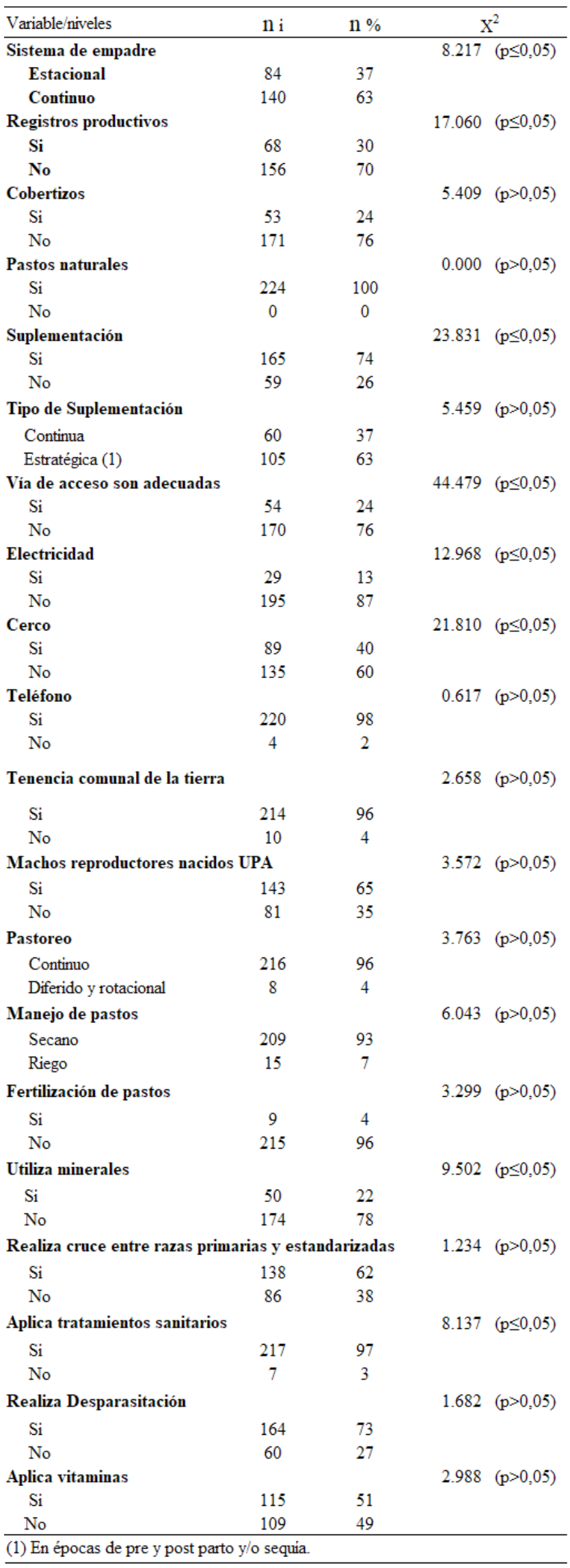

los $8,8( \pm 1,0)$ años $(\mathrm{P} \leq 0,05)$ y de las hembras a los 7,9 $( \pm 1,1)$ años $(\mathrm{P} \leq 0,05)$.

En el 65\% de las UPA se utilizan machos reproductores nacidos en la misma UPA; coincidiendo con Wolfinger (2012, p.47), quien reportó que los campesinos, al criar llamas, a menudo toman los sementales de sus propias manadas. Este manejo inadecuado consecuentemente tiende a generar elevada consanguinidad, que adquiere mayor relevancia en caso de los caracteres productivos; tal como lo declaró Vilela (2011, p.2) quien aseveró que debido a un ligero incremento de la consanguinidad como el 1\% dentro de la población puede causar pérdidas económicas a largo plazo por una reducción en la productividad animal. En cuanto a los registros de producción y reproducción, si bien es cierto que se utilizan, también es cierto que no son adecuados a lo requerido por la referencia; coincidiendo en parte con Gonzálvez (2010, p.46) quien concluyó que las granjas familiares de ganado vacuno no llevan a cabo registros de prácticamente ninguna actividad, coincidiendo con Vargas (2014, p.82,84) quien mencionó que los sistemas de producción caprino no llevan registros de producción ni de reproducción; estos resultados contradicen a Ascencio and Centeno (2013, p.1) quienes recomendaron que un sistema de producción debe guardar registro y estadísticas correspondientes.

\section{MANEJO de LA ALIMENTACIÓN}

El sistema de alimentación más utilizado es el pastoreo de forma continua en el 96\% de las UPA en estudio (Tabla IV); asemejándose a Valerio et al. (2010, p.333) quienes reportaron que la alimentación de ovejas y cabras se basa en un pastoreo continuo y diferido de pasturas naturales y bosques (94\%).

En el altiplano andino de Huancavelica las UPA basan su alimentación en pastos naturales al 100\%. La producción de pasturas se realiza en el 93\% de las UPA en secano y sin fertilización (96\%), lo que dificulta la disponibilidad de alimentos, especialmente en época de escasez de forrajes, limitando la vida útil de las pasturas; en concordancia con Cárdenas et al. (2015, p.168) quienes afirmaron que los compuestos de nitrógeno adquieren mayor importancia durante la época de reproducción y la estación seca del año, donde los bajos niveles de proteína en los pastizales limitan la actividad microbiana de los rumiantes, lo que afecta la digestibilidad y el consumo de forraje; coincidiendo también con Mendoza (2007, p.34) quien aseveró que las alpacas se alimentan exclusivamente del forraje disponible en los pastizales naturales.

Por otro lado, se observó una disminución en el diámetro de la fibra, aunque esta finura aparente podría deberse a las malas condiciones de alimentación de los animales en estudio en pasturas naturales (Quispe et al. 2009a, p.711).

El 74\% de las UPA ofrecen suplementación, continua $(37 \%)$ o estratégica $(63 \%)(\mathrm{P} \leq 0,05)$, siendo evidente la creciente predisposición a suplementar a los animales que conllevará a una mejor productividad; acorde a lo manifestado por Paucar et al. (2016, p.87) quienes concluyeron que la suplementación con en- 
silado tiene efecto sobre la ganancia de peso y es posible que también sobre la mortalidad en alpacas, por tanto la suplementación con ensilado en los meses de estiaje sería una buena alternativa para mantener el estado nutricional de las alpacas. Se está entendiendo con mayores raíces en la PUA que la suplementación nutricional es una buena alternativa para mejorar los índices reproductivos de la alpaca, ya que el consumo en pasturas nativas no satisface completamente los requerimientos nutricionales de las alpacas, y el déficit de forraje tiene un impacto directo en la fertilidad y tasas de mortalidad de las crías. El 51\% aplica vitaminas $(\mathrm{P} \leq 0.05)$ (Tabla IV); de acuerdo a lo reportado por Bravo y Alarcón $(2015$, p.455) quienes concluyeron que la suplementación nutricional es una buena alternativa para mejorar los índices reproductivos de la alpaca al evaluar el efecto de la suplementación nutritiva de machos y hembras durante la reproducción, utilizando productos comerciales para evaluar la calidad del semen, motilidad, concentración, espermatozoides vivos y el tiempo de reproducción. Asimismo, se utilizan minerales en el $20 \%$ de las UPA, encontrando un comienzo hacia la mejora nutricional, en vista que Cárdenas (2013, p.33) reportó que El 100\% de los productores no utilizan sales minerales.

\section{INTENSIFICACIÓN PRODUCTIVA Y ESTRUCTURA DEL REBAÑO}

Se reconoce que las causas principales antrópicas que afectan la productividad de algunas UPA son factores de manejo inapropiado; coincidiendo con Villalta et al. (2016, p.305) quienes destacaron el evidente sobrepastoreo que se observa y que da lugar al deterioro de las especies palatables, permitiendo la invasión de especies no deseables; en concordancia con Mendoza (2007, p.34) quien mencionó que en la actualidad se está desarrollando una mala práctica de manejo, como es la quema de pastizales, provocando un deterioro de las praderas. El uso irracional del agua contribuye a la degradación del suelo que, si continúa, puede llevar a la desertificación a corto y mediano plazo, lo que ocasiona graves problemas en la producción de pastos forrajeros; en consonancia con Alzérreca et al. (2001, p.1), quienes aseveran que las causas de la improductividad de algunas unidades productivas son factores de manejo inadecuados tales como la sobrecarga animal, el pastoreo continuo, el pastoreo excesivo, el mal manejo del agua y el uso de varias especies de ganado en el mismo nivel.

Respecto al componente animal, el 62\% de las UPA utilizan cruces entre razas primarias y estandarizadas, aunque estas últimas cada vez se utilizan con más frecuencia, principalmente como razas mejoradoras para cruces comerciales; contrariamente a lo encontrado por Mendoza (2007, p.34) quien aseveró que para el cruce de llamas se usan machos reproductores del mismo hato. El peso promedio al nacimiento es de 7,8 $\pm 1,0 \mathrm{~kg}(\mathrm{P} \leq 0,05)$ con un coeficiente de variabilidad de $13,1 \%$ con rangos que van desde 6 a $10 \mathrm{~kg}$, y de un total de 224 datos analizados (Figura 3), con diferencia estadística significativa $(\mathrm{p}<0,05)$ entre las cuatro provincias en estudio, dentro de un régimen de uso de pastos naturales, considerando que las UPA se desarrollan principalmente en tierras comunales; tal como lo reportó Quispe et al. (2007, p.1) quienes encontraron un peso promedio al nacimiento de 7,08 $\pm 0.06 \mathrm{~kg}$ en una muestra de 413 crías.

La edad y peso promedio al destete es de 7,7 $\pm 0,8$ meses y $25,2 \pm 1,1 \mathrm{~kg}$, respectivamente. En la provincia de Huancavelica los tuis presentan pesos al destete superiores, con un promedio de 25,9 $\pm 0,9$ / animal $(\mathrm{P} \leq 0,05)$. Estas diferencias pueden ser atribuidas a una mayor disponibilidad de área de pastoreo y una mayor diversidad en la vegetación.

Se encontró en las UPA una carga animal media de $0,8 \pm 0,1 \mathrm{UA} / \mathrm{ha} /$ año no existiendo diferencia estadística entre las provincias estudiadas $(p>0,05)$, dentro de un régimen de uso de pastos naturales, considerando que las UPA se desarrollan mayoritariamente en tierras comunales.

El cambio climático que induce la presencia de sequías prolongadas en los últimos años, sumado a la alta evaporación-transpiración causada por el aumento de la temperatura, contribuye a la degradación del suelo que, si continúa, puede llevar a la desertificación en el corto plazo a mediano y largo plazo, generando serios problemas en la producción de gramíneas forrajeras.

En términos productivos, la tasa de natalidad encontrada en el presente estudio es del 57,3 $\pm 2,7 \%$ $(\mathrm{P} \leq 0,05)$, similar a lo reportado por Gallegos (2013, p.255) quien determinó en el departamento de Puno una tasa de natalidad bruta para alpacas Huacaya de $59,6 \%$.

\section{MANEJO SANITARIO}

El 97\% de las UPA aplican tratamiento sanitario, observándose una tendencia mayor respecto a la aplicación de tratamientos en las estancias de la provincia de Huancavelica. El 73\% de las UPA aplican tratamientos con desparasitación mientras que el 51\% aplica vitaminas $(\mathrm{P} \leq 0,05)$. Se encontró una tasa de mortalidad promedio de 5,6 $\pm 1,4 \%$ en adultos y de $33,6 \pm 4,0 \%$ en crías $(\mathrm{P} \leq 0.05)$, destacándose niveles superiores en las UPA de la provincia de Angaraes 38,2 $\pm 2,3 \%$; similar a lo reportado por Fernández Baca $(2005$, p.26) quien aseveró que la mortalidad de las crías es considerada uno de los problemas de mayor impacto económico que enfrentan los productores de alpaca, ya que alcanzan cifras elevadas que en algunos años pueden superar el 50\%; contrariamente a lo reportado por Gallegos (2013, p.255) quien determinó en el departamento de Puno una tasa de mortalidad promedio para crías de alpacas Huacaya de 15,4\%. Dentro de las principales causas de mortalidad, destacan las muertes por enfermedades infecciosas como la enterotoxemia y neumonía, por enfermedades parasitarias como gastroenteritis verminosa y distomatosis, por depredadores e intoxicaciones; de manera similar a lo reportado por Mamani et al. (2009, p.5) quienes determinaron que entre las principales causas de mortalidad de alpacas del departamento de Puno se encuentran las enfermedades infecciosas en un 51,70\%. La escasez de instalaciones adecuadas para las crías y el diseño de corrales de descanso inadecuados favorecen la incidencia de enfermedades infecciosas y parasitarias, así como también la elevada mortalidad causada por depredadores. 
Es importante destacar que el $60 \%$ de los productores generalmente desconocen las causas de mortalidad, debido al manejo extensivo de los rebaños y a la falta de formación técnica con relación a aspectos sanitarios de la UPA.

\section{CONCLUSIONES}

Los sistemas de producción de las UPA del departamento de Huancavelica presentan heterogeneidad en su estructura productiva. Las UPA se concentran fundamentalmente en la provincia de Huancavelica, las que presentan productividad superior frente a los sistemas de las otras tres provincias del departamento de Huancavelica presentando una elevada dimensión promedio por unidad productiva de 170 cabezas y realizando un manejo más tecnificado frente al predominante sistema de producción tradicional extensivo. Las UPA analizadas presentan mayor uso de tierras comunales $(96 \%)$, uso frecuente de cercas sobre todo de piedras superpuestas, aunque no se diferencian significativamente en cuanto a carga animal (0,8 UA/ ha/año), suplementación en un 74\% que evidencia la creciente predisposición a suplementar a los animales entendiendo que conllevará a una mejor productividad, uso de pastos naturales $(100 \%)$, y mejor control sanitario (97\%) observándose una tendencia mayor, manejo óptimo de los parámetros reproductivos (11 hembras por macho). La crianza de alpacas a nivel comunal es prácticamente de subsistencia, caracterizada por infraestructuras inadecuadas, instalaciones de manejo deficientes, falta de planificación de la reproducción, suplementación deficiente con reducidas tasas de fertilidad y elevadas pérdidas neonatales que difícilmente pueden ser analizadas por carencia de registros productivos y sanitarios; y que en términos generales constituyen factores técnicos y estructurales que limitan el desarrollo eficiente y sostenible del sistema de producción de alpacas en el departamento de Huancavelica..

\section{AGRADECIMIENTOS}

Al Centro de Producción e Investigación de Camélidos Sudamericanos "Lachocc" de la Universidad Nacional de Huancavelica, por permitir el uso de sus instalaciones para realizar esta investigación.

A las familias Salvatierra, Marmolejo, Quispe y Taype de las comunidades campesinas de Angaraes, Castrovirreyna, Huancavelica y Huaytará, respectivamente quienes me abrieron las puertas de sus casas y de sus corazones, compartiendo sus saberes y experiencias, pastores y pastoras quienes me permitieron tomar fotos y filmar sus prácticas de manejo en las unidades productivas.

\section{BIBLIOGRAFÍA}

Aguirre, F, Matta, S, \& Montero, C 2011 , 'Producción comercial de fibra fina de alpaca mediante tecnologías de reproducción asistida y crianza semi-intensiva', [Tesis]. UPC. Perú, pp. 12, 48-49.

Alzérreca, H, Luna, D, Prieto, G, Cardozo, A \& Céspedes, J 2001, 'Estudio de la capacidad de carga en bofedales para la cría de alpacas en el sistema T.D.P.S', PNUD-AIGACAA. Bolivia, pp.98.
Allende, S 2008, 'Evaluación del estado nutricional de llamas madres y crías en pastoreo natural', Tesis IZ, UNSAAC, p. 21 .

Ascencio, B \& Centeno, R 2013, 'Sistema integral de gestión de la producción de fibra de los camélidos sudamericanos en las asociaciones ganaderas del distrito de Ajoyani, provincia de Carabaya, Puno-Perú', Revista Comuni@cción V.1, N. 1, ISSN 2219-7168, p. 1.

Bravo, W \& Alarcón, V 2015, 'La Influencia de Suplementos Nutritivos en la Calidad de Semen y Fertilidad de la Alpaca', Revista de Investigaciones Altoandinas, 2015, Vol 17 №3, pp.453-456.

Buritica, J 2010, 'Caracterización de la producción regional de fibra de alpaca a la postcosecha en el corredor económico central de Huancavelica', Escuela técnica superior de ingenieros agrónomos. UPNA, España, p. 13

Cárdenas, J, Paulino, M, Lopes, S, Silva, A, Barros, L, \& Valente, É 2015, 'Desempeño productivo, consumo y digestibilidad de terneras lactantes criadas en pastoreo suplementadas con diferentes niveles de proteína ruta', Archivos de zootecnia, 64(246).

Cárdenas, J 2013, 'Bioseguridad en la crianza de alpacas en el distrito de Santa Ana-Castrovirreyna-Huancavelica', [Tesis]. EAPZUNH, pp. 6, 33

Carné, S, Roig, N, \& Jordana, J 2007, 'La cabra blanca de Rasquera: Caracterización estructural de las explotaciones', Archivos de zootecnia, 56(213), p.43.

Castaldo, A, Acero, R, Perea, J, Martos, J, Dominech, V, Pamio, J, \& García, A 2006, 'Tipología de los sistemas de producción de engorde bovino en la Pampa Argentina', Archivos de Zootecnia. 55: 184.

Celorio, S, Argüello, S, Barquín, F. Crespo, M, Chomón, N, Cimadevilla, Calderón, L, \& Cañón, J 2011, 'Caracterización estructural de las explotaciones de ganado bovino de raza Pasiega', Archivos de zootecnia, 60(231), 409.

Cochi, N, Prieto, G, Dangles, O, Rojas, A, Ayala, C, Condori, B, \& Cazasola, J 2014, 'Metodología para evaluar el potencial productivo y la dinámica socioecológica de la ganadería en bofedales altoandinos', Revista Ecología en Bolivia, 49(3): 122. ISSN 1605-2528.

Fairfield, T 2006, 'The politics of livestock sector policy and the rural poor in Peru', Pro-Poor Livestock Policy Initiative, Food and Agriculture Organization. PPLPI Working Paper № 32, Rome: FAP. p.32.

Fernández Baca, S 2005, 'Situación actual de los camélidos sudamericanos en Perú', Proyecto de Cooperación Técnica en apoyo de la crianza y aprovechamiento de los Camélidos Sudamericanos en la Región Andina TCP/RLA/2914. Organización de las Naciones Unidas para la Agricultura y la Alimentación, p.26.

Gallegos, R 2013, 'Índices productivos de alpacas del Centro de Investigacióny Producción La Raya'. Revista de Investigaciones Altoandinas, 2013, Vol 15 N $^{\circ}$ 2: 255-262.

García, E \& Otto, M 2015, 'Caracterización ecohidrológica de humedales alto-andinos usando imágenes de satélite multitemporales en la cabecera de cuenca del río santa, Ancash, Perú', Ecología Aplicada, UNALM, 14(2), 2015. ISSN 1726-2216, pp.115-125.

Gaspar, G, Mesías, D, Escribano, S, \& Pulido, G 2009, 'Evaluación de la sostenibilidad en explotaciones de dehesa en función de su tamaño y orientación ganadera', ITEA Vol. 105(2), 117-141.

Gómez, N 2013, 'Caracterización estructural, morfológica y genética de la población de cabras autóctonas de la región Apurímac del Perú', [Tesis Doctoral]. Facultad de Veterinaria. UNAB, Barcelona, España, pp. 267.

Gonzálvez, J 2010, 'Caracterización estructural de las explotaciones de vacuno lechero de la comunidad valenciana para la implantación de un sistema de calidad agroalimentaria "GLOBAL G.A.P. ', pp.72.

Hinojosa, R \& Ruiz, J 2003, 'Evaluación del comportamiento sexual y efecto de la edad materna sobre la fertilidad en alpacas Huacaya con empadre controlado', III Congreso Mundial sobre Camélidos y I Taller Internacional de DECAMA. Potosí, Bolivia.

INEI, 2012, 'Resultados Definitivos: IV Censo Nacional Agropecuario 2012', Lima, Perú. 
Laura, J 2015, 'Caracterización de los sistemas de producción en comunidades alpaqueras de la provincia Pacajes', [Tesis Doctoral], La Paz, Bolivia, pp.169

Mamani, P, Condemayta, C, \& Calle, Ch 2009, 'Causas de mortalidad de alpacas en tres principales centros de producción ubicados en puna seca y húmeda del departamento de Puno', REDVET. Revista electrónica de Veterinaria. ISSN: 1695-7504 2009 Vol. 10, № 8.

Mendoza, B 2007, 'Factores que intervienen en los sistemas de producción sustentables de los pequeños rumiantes y camélidos', In: Memorias del III Simposio Latinoamericano sobre Crianza en Forma Sustentable de Pequeños Rumiantes y Camélidos Sudamericanos, Abancay-Perú, pp. 26-39.

Paucar, R, Aquino, H, Contreras, J, Caso, E, \& Ruiz, A 2016, 'Efecto de la suplementación con ensilado (Festuca dolichophylla, Avena sativa y Vicia sativa) sobre la ganancia de peso y mortalidad en alpacas adultas (Vicugna pacos)', Rev. Complutense de Ciencias Veterinarias, 2016 10(1):83-88.

Parodi, N201 1, Principales problemas en la calidad de la fibra de al paca que limitan la comercialización de prendas de vestir en el mercado francés', Revista de Ciencias Empresariales. Vol. 2 № 2, pp.21-29.

Quina, E 2015, 'Diagnóstico de la crianza y caracterización fenotípica de las llamas k' ara (Lama glama) en Marcapomacocha', [Tesis], UNALM, Junín, Perú.

Quispe, E, Alfonso, L, Flores, A, Guillen, H, \& Ramos, Y 2009a, 'Bases para un programa de mejora de alpacas en la región altoandina de Huancavelica-Perú', Archivos de Zootecnia, 58 (224): 705-716.

Quispe, E, Rodríguez, T, Iñiguez, L, \& Mueller, J 2009b, 'Producción de fibra de alpaca, llama, vicuña y guanaco en Sudamérica', Recursos genéticos animales, 45, 1-14.

Quispe, E, Flores, A, Alfonso, L, \& Galindo, A 2007, ‘Algunos aspectos de la fibra y peso vivo de alpacas Huacaya de color blanco en la región de Huancavelica', APPA - ALPA - Cusco, Perú, 2007.

Saadoun, A \& Cabrera, M 2008, 'A review of the nutritional content and technological parameters of indigenous sources of meat in South America', Meat Science 80: 570-581.

Salvá, B, Zumalacárregui, J, Figueira, A, Osorio, M, \& Mateo, J 2009, 'Nutrient composition and technological quality of meat from alpacas reared in Peru', Meat Science, 82: 450455.
Santos, J, Muñoz, A, Juez, P, \& Cortinas, P 2004, 'Diseño de encuestas para estudios de mercado', Primera reimpresión. Editorial Centro de Estudios Ramón Areces, S.A., pp.167-183

Squeo, F, Warner, B, Aravena, R, \& Espinoza, D 2006, 'Bofedales: High altitude peatlands of the central Andes', Revista Chilena de Historia Natural,79: 245-255.

Sulbarán, L, Drescher, K, Martínez, N, Colmenares, O, \& Ricca, R 2008, 'Technical diagnosis of the production system with dual-purpose bovine in the hilly area in Guárico state, Venezuela', Zootecnia Tropical, 26(2): 79-85.

Valerio, D, García, A, Acero, R, Perea, J, Tapia, M, \& Romero, M 2010, 'Caracterización estructural del sistema ovino-caprino de la región noroeste de República Dominicana', Archivos de zootecnia, 59(227), 333-343.

Valerio, D, García, A, Perea, J, Acero, R, \& Gómez, G 2009, 'Caracterización social y comercial de los sistemas ovinos y caprinos de la región noroeste de República Dominicana', Interciencia, 34: 637-644.

Vargas, J 2014, 'Caracterización de los sistemas de producción en la comunidad de Higuerayoc, provincia Sud Cinti del departamento de Chuquisaca (Doctoral dissertation) ', La paz, Bolivia.

Vargas, E 2007, 'Evaluación del sistema de producción ganadero de la comunidad Kjari Alta, Caquiaviri provincia Pacajes', (Doctoral dissertation), Bolivia

Vilela, J 2011, 'Consanguinidad y su importancia en el mejoramiento genético de la alpaca', Revista SIRIVS. UNMSM.

Villalta, R, Zapana, P, Zapana, L, Araoz, B, \& Escobar, M 2016, 'Evaluación de pastos y capacidad de carga animal en el fundo "Carolina" de la Universidad Nacional del Altiplano-Puno, Perú', Revista de Investigaciones Altoandinas, 2016; Vol 18 N$^{\circ} 2: 303-310$.

Wolfinger, B 2012, 'Characterization of the production system of llamas and description of breeding strategies of smallholders in the Central Peruvian Andes', [Tesis], University of Natural Resources and Life Sciences Vienna. Pasco, Perú. 